\title{
One and Two-photon Physics with Relativistic Heavy Ions
}

\author{
C.A. Bertulani \\ Department of Physics, Brookhaven National Laboratory, Upton, NY 11973-5000, USA
}

(October 26, 2018)

The physics of peripheral collisions with relativistic heavy ions (PCRHI) is reviewed. One- and two-photon processes are discussed.

PACS: 25.20.Lj, 25.60.-t,25.75.-q,25.80.-e

\section{PERIPHERAL COLLISIONS}

In peripheral collisions of relativistic heavy ions (PCRHI) [1] the number of equivalent photons with energy $\omega, n(\omega)$, has been calculated classically [2], and quantum-mechanically [3]. It has been shown [3] that for the electric dipole multipolarity, E1, the equivalent photon number, $n_{E 1}(\omega)$, coincides with the one deduced classically [2] for very forward scattering. E2-photons are more abundant at low energies. But, in the extreme relativistic collisions the equivalent photon numbers for all multipolarities agree, i.e, $n_{E 1}(\omega) \sim n_{E 2}(\omega) \sim n_{M 1}(\omega) \sim$

.... The cross sections for one- and two-photon processes depicted in figure 1 are given approximately by

$$
\sigma_{X}=\int d \omega \frac{n(\omega)}{\omega} \sigma_{X}^{\gamma}(\omega), \text { and } \sigma_{X}=\int d \omega_{1} d \omega_{2} \frac{n\left(\omega_{1}\right)}{\omega_{1}} \frac{n\left(\omega_{2}\right)}{\omega_{2}} \sigma_{X}^{\gamma \gamma}\left(\omega_{1}, \omega_{2}\right),
$$

where $\sigma_{X}^{\gamma}(\omega)$ is the photon-induced cross section for the energy $\omega$, and $\sigma_{X}^{\gamma \gamma}\left(\omega_{1}, \omega_{2}\right)$ is the two-photon cross section. For one-photon processes, e.g., Coulomb fragmentation, $\sigma_{X}^{\gamma}(\omega)$ is localized in a small energy interval and one gets a cross section in the form $\sigma=A \ln \gamma_{c}+B$, where $A$ and $B$ are coefficients depending on the system. The $\ln \gamma_{c}$ factor is due to the equivalent photon number (EPA), $n(\omega)$, which is approximately independent of $\omega$ in the integral range of interest. As for the two-photon processes, besides the $\ln ^{2} \gamma_{c}$ from $n_{1}$ and $n_{2}$, a third $\ln \gamma_{c}$ arises from the integral over $\omega_{1}\left(\omega_{1}\right.$ and $\omega_{2}$ are related by energy conservation). Note that here we used $\gamma_{c}$ of a HI-collider, so that $\gamma=2 \gamma_{c}^{2}-1$, with $\gamma_{c}$ the collider Lorentz gamma factor $\left(\gamma_{c} \sim 100\right.$ for RHIC/BNL). 
(a)
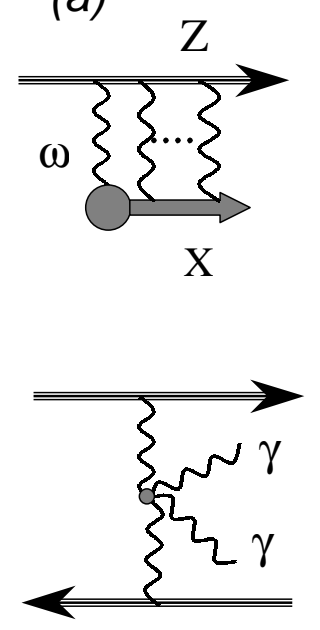

(d) (b)

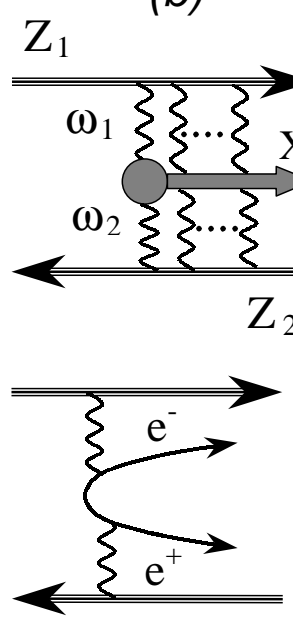

(e) (c)
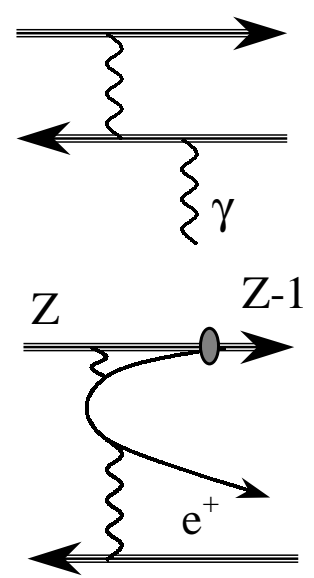

(f)

FIG. 1. PCRHI processes: (a) one-photon, (b) two-photon, (c) Bremsstrahlung, (d) Delbrück scattering, (e) pair-production, and (f) pair-production with capture.

\section{BREMSSTRAHLUNG AND DELBRÜCK SCATTERING}

Bremsstrahlung (fig. 1c) is a minor effect in PCRHI 四. The cross section is proportional to the inverse of the square mass of the ions. Most photons have very low energies. For $10 \mathrm{MeV}$ photons the central collisions (CCRHI) deliver $10^{6}$ more photons than the PCRHI [5]. For a collider the Bremsstrahlung differential cross section is given by $d \sigma_{\gamma} / d \omega=16 Z^{6} \alpha^{3}\left(3 \omega A^{2} m_{N}^{2}\right)^{-1} \ln (\gamma / \omega R)$, where $m_{N}$ is the nucleon mass, $\gamma=2 \gamma_{c}^{2}-1$, where $\gamma_{c}$ is the collider Lorentz gamma factor $\left(\gamma_{c} \sim 100\right.$ for RHIC/BNL), and $R$ is the nuclear dimension $\left(R \sim 2.4 \times A^{1 / 3} \mathrm{fm}\right)$ [5].

For very low energy photons $(\omega \sim 100 \mathrm{eV})$ the whole set of particles in a bunch act coherently and a great number of Bremsstrahlung photons are produced. This has been proposed as a tool for monitoring the bunch dimensions in colliders [6].

Delbruck scattering $\left(\gamma^{*}+\gamma^{*} \longrightarrow \gamma+\gamma\right)$ involves an additional $\alpha^{2}$ as compared to pair production and has never been possible to study experimentally. The cross section is about $50 \mathrm{~b}$ for the LHC [5] and the process is dominated by high-energy photons, $E_{\gamma} \gg m_{e}$. A study of this process in PCRHI is thus promising if the severe background problems arising 
from CCRHI can be eliminated. No experiments of Bremsstrahlung or Delbrück scattering in PCRHI have been performed so far. The total cross section for Delbrück scattering $\left(\omega \gg m_{e}\right)$ in colliders is given by $\sigma=2.54 Z^{4} \alpha^{4} r_{e}^{2} \ln ^{3}\left(\gamma / m_{e} R\right)$, where $r_{e}=e^{2} / m_{e}$ is the classical electron radius [5].

\section{ATOMIC IONIZATION}

The cross sections for atomic ionization are very large, of order of kilobarns, increasing slowly with the logarithm of the RHI energy. For a fixed target experiment using naked projectiles one gets [四: $\sigma_{I}=Z_{P}^{2} r_{e}^{2}\left(Z_{T} \alpha\right)^{-2}\left[1.8 \pi+9.8 \ln \left(2 \gamma / Z_{T} \alpha\right)\right]$, which decreases with the target charge $Z_{T}$. This is due to the increase of the binding energy of $K$-electrons with the atomic charge. The first term is due to close collisions assuming elastic scattering of the electron off the projectile, while the second part is for distant collisions, with impact parameter larger than the Bohr radius. Recently, Baltz [9] has shown that the numerical factors in the equation above should be replaced by $1.8 \rightarrow 1.74-1.83$ and $9.8 \rightarrow 7.21$, respectively, when one includes higher order terms in the perturbation series. The probability to eject a $K$-electron is much larger than for other atomic orbitals. Recent experiments have reported ionization cross sections for $\mathrm{Pb}^{81+}(33 \mathrm{TeV})$ beams on several targets [8]. In this case, the role of projectile and target are exchanged in the previous equation. In figure 2 we show the results of this equation (dashed line) compared to the experimental data. Since the targets are screened by their electrons, the discrepancy is expected. Even the

most detailed calculations by Anholt and Becker [10] (solid line) or of Baltz [9] yield larger cross sections than the experimental data. 


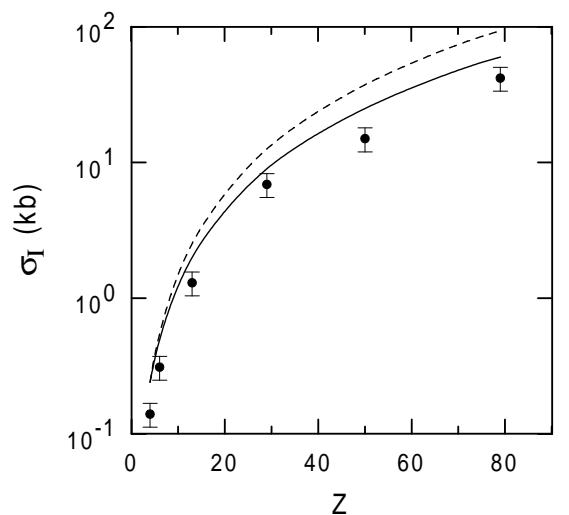

FIG. 2. Atomic ionization cross sections for $\mathrm{Pb}^{81+}(33 \mathrm{TeV})$

beams on several targets [8].

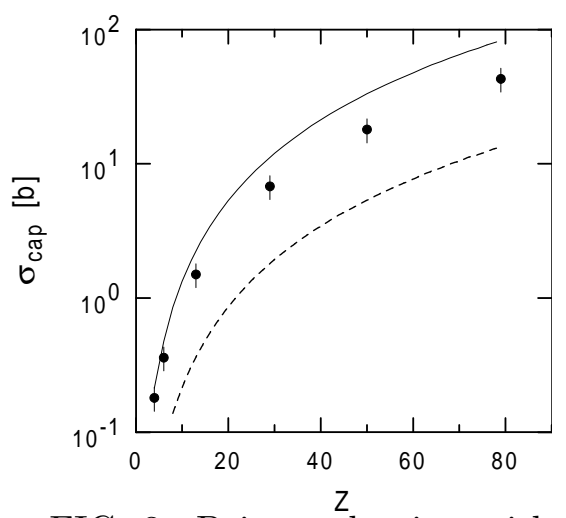

FIG. 3. Pair production with capture for $\mathrm{Pb}^{82+}(33 \mathrm{TeV})$ beams on several targets [8].

Non-perturbative calculations, solving the time dependent Dirac equation exactly, were first performed by Giessen and Oak Ridge groups [12,13]. The main problem is to adequately treat the several channels competing with the ionization process, specially for atoms with more than one electron. Also, the effects of screening (static and dynamical) are hard to calculate. On the experimental side, there are little data available for a meaningful comparison with theory.

\section{FREE AND BOUND-FREE ELECTRON-POSITRON PAIR PRODUCTION}

It has been demonstrated long time ago [14 that to leading order in $\ln \gamma$, the $e^{+} e^{-}$-pair production in PCRHI is given by $\sigma=(28 / 27 \pi) Z_{P}^{2} Z_{T}^{2} r_{e}^{2} \ln ^{3}(\gamma / 4)$. A renewed interest in this process appeared with the construction of relativistic heavy ion accelerators. For heavy ions with very large charge (e.g, lead, or uranium) the pair production probabilities and cross sections are very large. They cannot be treated to first order in perturbation theory [⿴囗⿴囗十), and are also difficult to calculate. This resulted in a great amount of theoretical studies [15].

Replacing the Lorentz compressed electromagnetic fields by delta functions, and working with light cone variables, one has developed more elaborate calculations [16], recently. The latest debate around the subject is the proper treatment of Coulomb distortion of the lepton wavefunctions, and of production of n-pairs [16]. 
An important phenomenon occurs when the electron is captured in an atomic orbit of the projectile, or of the target. In a collider this leads to beam losses each time a charge modified nucleus passes by a magnet downstream [5]. A striking application of this process was the recent production of antihydrogen atoms using relativistic antiproton beams [17]. Here the positron is produced and captured in an orbit of the antiproton. Early calculations for this process used perturbation theory [10,18,4]. Some authors use nonperturbative approaches, e.g., coupled-channels calculations [15]. Initially some discrepancy with perturbative calculations were found, but later it was shown that non-perturbative calculations agree with the perturbative ones at the $1 \%$ level (see, e.g., first reference of [16]).

The expression $\sigma=3.3 \pi Z^{8} \alpha^{6} r_{e}^{2}[\exp (2 \pi Z \alpha)-1]^{-1}[\ln (0.681 \gamma / 2)-5 / 3]$ for pair production with electron capture in PCRHI was obtained in ref. 4 . The term $[. . .]^{-1}$ is the main effect of the distortion of the positron wavefunction. It arises through the normalization of the continuum wavefunctions which accounts for the reduction of the magnitude of the positron wavefunction near the nucleus where the electron is localized (bound). Thus, the greater the $Z$, the less these wavefunctions overlap. The above equation predicts a dependence of the cross section in the form $\sigma=A \ln \gamma+B$, where $A$ and $B$ are coefficients depending on the system. This dependence was used in the analysis of the experiment in ref. [8]. In recent calculations, attention was given to the correct treatment of the distortion effects in the positron wavefunction [19]. In figure 3 we show the recent experimental data of ref. [8] compared to the above equation and recent calculations (second reference of [19]). These calculations also predict a $\ln \gamma$ dependence but give larger cross sections than in ref. [4]. The comparison with the experimental data is not fair since atomic screening was not taken into account. When screening is present the cross sections will always be smaller up to a factor of 2 [4]. The conclusion here is that pair production with electron capture is a process which is well treated in first order perturbation theory. Again, the main concern is the correct treatment of distortion effects (multiphoton scattering) [19].

The production of para-positronium in heavy ion colliders was calculated [20]. The 
cross section at RHIC is about $18 \mathrm{mb}$. This process is of interest due to the unusual large transparency of the parapositronium in thin metal layers.

\section{RELATIVISTIC COULOMB EXCITATION AND FRAGMENTATION}

Relativistic Coulomb excitation is becoming a popular tool for the investigation of the intrinsic nuclear dynamics and structure of the colliding nuclei, specially important in reactions involving radioactive nuclear beams [21,22]. Coulomb excitation and dissociation of such nuclei are common experiments in this field [1,23]. The advantage is that the Coulomb interaction is very well known. The real situation is more complicated since the contribution of the nuclear-induced processes cannot be entirely separated in the experimental data. The treatment of the dissociation problem by nuclear forces is very model dependent, based on eikonal or multiple Glauber scattering approaches [22,24]. Among the uncertainties are the in-medium nucleon-nucleon cross sections at high-energies, the truncation of the multiple scattering process and the separation of stripping from elastic dissociation of the nuclei [25]. Nonetheless, specially for the very weakly-bound nuclei, re

lead to very exciting new results [22,24].

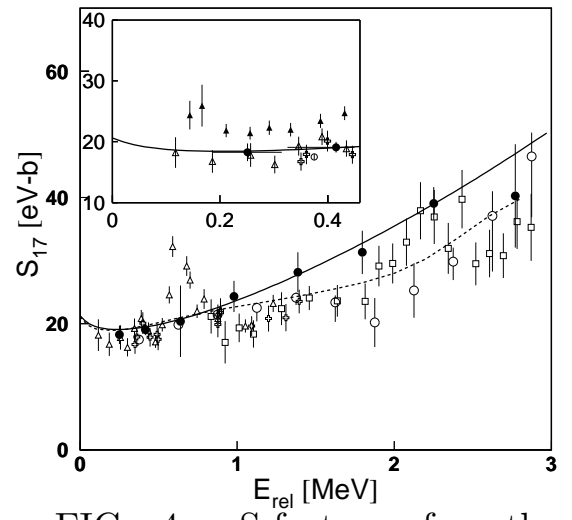

FIG. 4. S-factors for the

${ }^{7} B e(p, \gamma)^{8} B$ reaction.

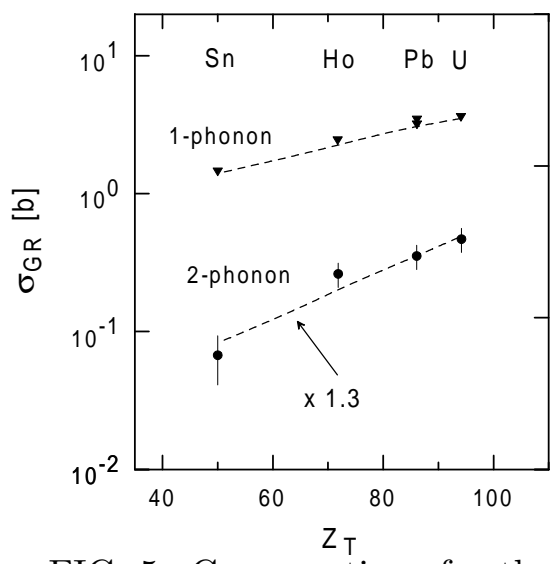

FIG. 5. Cross sections for the excitation of the GDR and the DGDR.

In the Coulomb breakup of weakly-bound nuclei one speculates if the reaction proceeds via a single or multiple photon-exchange between the projectile and the target. In the first 
case, perturbation theory gives a direct relation between the data and the matrix element for electromagnetic dissociation. Such matrix elements are the clearest probes one can get about the nuclear structure of these nuclei. In the second case, often called by postacceleration effects [24], one has to perform a non-perturbative treatment of the reaction what complicates the extraction of the electromagnetic (mainly E1) matrix elements. One expects to learn if the Coulomb-induced breakup proceeds via a resonance or by the direct dissociation into continuum states [24]. There is a strong ongoing effort to use the relativistic Coulomb excitation technique also for studying the excitation of bound excited states in exotic nuclei, to obtain information on gamma-decay widths, angular momentum, parity, and other properties of hitherto unknown states [23].

Radiative capture reactions are known to play a major role in astrophysical sites, e.g., in a pre-supernova [26]. Some of these reactions, like for example, ${ }^{7} B e(p, \gamma)^{8} B$, can be studied via the inverse photo-dissociation reaction ${ }^{8} B(\gamma, p)^{7} B e$. One often uses the astrophysical S-factor, defined by $S(E)=E \sigma(E) \exp [-2 \pi \eta(E)]$, where $\eta(E)=Z_{1} Z_{2} e^{2} / \hbar \sqrt{2 \mu_{12} E}$, where $E$ is the relative kinetic energy of the two nuclei. The matrix elements involved in the dissociation processes are the same as those involved in the absorption by real photons [4]. One of the experiments using this technique was performed at the GSI/Darmstadt [27]. The S-factor obtained in this experiment is shown in figure 4 as solid circles.

A giant dipole resonance (GDR) occurs in nuclei at energies of 10-20 MeV. Assuming that these are harmonic vibrations of protons against neutrons, one expects that DGDRs (Double Giant Dipole Resonances), i.e., two giant dipole vibrations superimposed in one nucleus, will have exactly twice the energy of the GDR [4,21]. A series of experiments at the GSI/Darmstadt has obtained energy spectra, cross sections, and angular distribution of fragments following the decay of the DGDR. It was shown that the experimental cross sections are about $30 \%$ bigger than the theoretical ones. This is shown in figure 5 where the cross sections for the excitation of 1-phonon (GDR), $\sigma_{1} \sim 2 \pi S \ln \left[2 \gamma A_{T}^{1 / 3}\left(A_{P}^{1 / 3}+A_{T}^{1 / 3}\right)^{-1}\right]$, while for the 2-phonon state it is $\sigma \sim S^{2}\left(A_{P}^{1 / 3}+A_{T}^{1 / 3}\right)^{-2}$, where $S=5.45 \times 10^{-4} Z_{P}^{2} Z_{T} N_{T} A_{T}^{-2 / 3}$ mb. The dashed lines of figure 5 are the result of more elaborate calculations [21]. The 
GSI experiments are very promising for the studies of the nuclear response in very collective states. One should notice that after many years of study of the GDRs and other collective modes, the width of these states are still poorly explained theoretically, even with the best microscopic approaches known sofar. The extension of these approaches to the study of the width of the DGDRs will be helpful to improve such models.

In colliders it was shown that the mutual Coulomb excitation of the ions (leading to their simultaneous fragmentation) is a powerful tool for beam monitoring [28]. A recent measurement at RHIC by Sebastian White and collaborators [29], using the Zero Degree Calorimeter to measure the neutron decay of the reaction products, has proved the feasibility of the method. The theoretical prediction of about $3 \mathrm{~b}$ for this process, agrees quite well with the experimental results.

The DGDR contributes only to about $10 \%$ of the total fragmentation cross section induced by Coulomb excitation with relativistic heavy ions. The main contribution arises from the excitation of a single GDR, which decays mostly by neutron emission. This is also a potential source of beam loss in relativistic heavy ion colliders [1], and an important fragmentation mode of relativistic nuclei in cosmic rays.

\section{MESON AND HADRON PRODUCTION}

The production of heavy lepton pairs $\left(\mu^{+} \mu^{-}\right.$, or $\left.\tau^{+} \tau^{-}\right)$, or of meson pairs (e.g., $\pi^{+} \pi^{-}$) can be calculated using the second of equation (1). One just needs the cross sections for $\gamma \gamma$ production of these pairs. Since they depend on the inverse of the square of the particle mass [5], the pair-production cross sections are much smaller in this case. The same applies to single meson production by $\gamma \gamma$ fusion. The $\gamma \gamma$ cross section is given by $\sigma_{\gamma \gamma \rightarrow M}=8 \pi^{2}(2 \mathrm{~J}+$ 1) $\Gamma_{M \rightarrow \gamma \gamma} \delta\left(W^{2}-M^{2}\right) / M$, where $J, M$, and $\Gamma_{M \rightarrow \gamma \gamma}$ are the spin, mass and two-photon decay width of the meson, $W$ is the c.m. energy of the colliding photons [5]. In ref. [4] the following equation was obtained for the production of mesons with mass $M$ in HI colliders: $\sigma=Z^{4} \alpha^{2}\left(128 \Gamma_{\gamma \gamma} / 3 M^{3}\right) \ln ^{3}(2 \gamma \delta / M R)$, where $\delta=0.681 \ldots$ Later [30] it is shown that a more 
detailed account of the space geometry of the two-photon collision is necessary, specially for the heavier mesons.

A careful study of the production of meson pairs and single mesons in PCRHI was performed recently in ref. [31] (see also, [34]). In table I we show the magnitude of the cross sections for single meson production at RHIC and at LHC [31]. Also shown are the cross sections due to difractive processes (pomeron-pomeron exchange). We see that they are several orders of magnitude smaller than those from $\gamma \gamma$ fusion. The cross sections for the production of $\eta_{c}, \eta_{c}^{\prime}$ and $\eta_{b}$ are very small due to their higher masses. Similar studies have been done for meson production in $\gamma$-nucleus interactions. Particles like $\Delta, \rho, \omega, \phi, J / \Psi$, etc, can be produced in this way [32]. However, the one-photon exchange processes are more effective in the production of mesons in PCRHI. One considers the interaction between the photon from one nucleus with a Pomeron from another. These photon-Pomeron interactions were calculated in ref. [35] and are shown in table II.

The possibility to produce a Higgs boson via $\gamma \gamma$ fusion was suggested in ref. [33]. The cross sections for LHC are of order of 1 nanobarn, about the same as for gluon-gluon fusion. But, the two-photon processes can also produce $b \bar{b}$ pairs which create a large background for detecting the Higgs boson. A good review of these topics was presented in ref. [32].

The excitation of a hadron in the field of a nucleus is another useful tool to study the properties of hadrons. It has been used for example to obtain the lifetime of the $\Sigma_{0}$ particle by measuring the (M1) excitation cross section for the process $\gamma+\Lambda \rightarrow \Sigma_{0}$ [36]. The vertex $\gamma \rightarrow 3 \pi$ has been investigated [37] in the reaction of pion pair production by pions in the nuclear Coulomb field: $\pi^{-}+Z \rightarrow \pi^{-}+\pi^{0}+Z$. Also, the $\pi^{-}$polarizability has been studied in the reaction $\pi^{-}+Z \rightarrow \pi^{-}+\gamma+Z$ [38]. Other unexplored possibilities includes the excitation of a nucleon to a $\Delta$-particle in the field of a heavy nucleus in order to disentangle the $M 1$ and $E 2$ parts of the excitation.

As for meson-production in PCRHI there are several planned experiments at RHIC (see the contribution of Spencer Klein to these proceedings), as well as for the future LHC [39]. These machines were designed for study hadronic processes. But, as have shown in this brief 
review, they can also be used for studying very interesting phenomena induced in peripheral collisions.

\section{ACKNOWLEDGMENT(S)}

The author is a fellow of the John Simon Guggenheim Memorial Foundation. This

work has been authored under Contract N0. DE-AC02-98CH10886 with the DOE/USA and contract No. 41.96.0886.00 with the PRONEX/Brazil.

[1] C.A. Bertulani and G. Baur, Physics Today, March 1994, p. 22.

[2] E. Fermi, Z. Physik 29, 315 (1924); K. F. Weizsacker, Z. PhysikE, 612 (1934); E. J. Williams, Phys. Rev. 45, 729 (1934).

[3] C.A.Bertulani and G. Baur, Nucl. Phys. A442 (1985) 73.

[4] C.A. Bertulani and G. Baur, Physics Reports 163 (1988) 299.

[5] G. Baur and C.A. Bertulani, Z. Phys. A330 (1988) 77; C.A. Bertulani and G. Baur, Nucl. Phys. A505 (1989) 835.

[6] I.F. Ginzburg, G.L. Kotkin, S.I. Polityko and V.G. Serbo, Phys. Rev. Lett. 68 (1992) 788; Phys. Lett. B286 (1992) 392; Z. Phys. C60 (1993) 737; e-print hep-ph/9512310

[7] J. Eichler and. Meyerhof, Relativistic Atomic Collisions (Academic Press, San Diego, 1995); R. Anholt and H. Gould, in Advances in Atomic and Molecular Physics (Academic Press, San Diego, 1986), p. 315.

[8] H.F. Krause et al., Phys. Rev. Lett. 80 (1998) 1190.

[9] A.J. Baltz, physics/0102045; see also, A.B. Voitkov, C. Müller, and N. Grün, Phys. Rev. A62, $062701(2000)$ 
[10] R. Anholt and U. Becker, Phys. Rev. A36 (1987) 4628.

[11] A.J. Baltz, M.J. Rhoades-Brown and J. Weneser, Phys. Rev. A44 (1991) 5668; 47 1993) 344; 48 (1993) 2002; 50 (1994) 4842

[12] U. Becker, N. Grün and W. Scheid, J. Phys. B16 (1983) 1967; U. Becker, PhD. Thesis, Giessen (1986).

[13] C. Bottcher and M.R. Strayer, Phys. Rev. Lett. 54 (1985) 669.

[14] W.H. Furry and J.F. Carlson, Phys. Rev. 44 (1933) 238; L.D. Landau and E.M. Lifshitz, Phys. Zs. Sowjet, 6 (1934) 244; H.J. Bhabha, Proc. R. Soc. London Ser. A152 (1935) 559; Y. Nishina, S. Tomonaga and M. Kobayashi, Sci. Pap. Phys. Chem. Res. 27 (1935) 137.

[15] C. Bottcher and M.R. Strayer, Phys. Rev. D39 (1989)1330; J. Phys. G16 (1990) 975; Phys. Lett. B237 (1990) 175; G. Baur, Phys. Rev. D41 (1990) 3535; Phys. Rev. A42 (1990) 5736; M.J. Rhoades-Brown and J. Weneser, Phys. Rev. A4 (1991) 33; C. Best, W. Greiner and G. Soff, Phys. Rev. A46 (1992) 261; M. Vidović, M. Greiner, C. Best and G. Soff, Phys. Rev. C47 (1993) 2308; K. Hencken, D. Trautmann and G. Baur, Phys. Rev. A51 (1995) 998; A51 (1995) 1874;

[16] A.J. Baltz, Phys. Rev. Lett. 78 (1997) 1231; A.J. Baltz and L. McLerran, Phys. Rev. C58 (1998) 1679; B. Segev and J.C. Wells, Phys. Rev. A57 (1998) 1849; D.Yu. Ivanov, A. Schiller, V.G. Serbo, Phys. Lett. B454 (1999) 155; U. Eichmann, J. Reinhardt, S. Schramm and W. Greiner, Phys. Rev. A59 (1999) 1223; A.J. Baltz, F. Gelis, L. McLerran and A. Peshier, nucl-th/0101024; R.N. Lee and A.J. Milstein, hep-ph/0103212

[17] G. Baur et al., Phys. Lett. B368 (1996) 351; G. Blanford et al., Phys. Rev. Lett. 80 (1998) 3037.

[18] U. Becker, J. Phys. B20 (1987) 6563; K. Momberger, N. Grün and W. Scheid, Z. Phys. D18 1991) 133; K. Rumrich, G. Soff and W. Greiner, Phys. Rev. A47 1993) 215; K. Momberger, A. Belkacem and A.H. Sorensen, Europhys. Lett. 32 (1995) 401. 
[19] H. Meier et al., e-print nucl-th/0008020; C.A. Bertulani and D. Dolci, Nucl. Phys. A683 (2001) 635

[20] G. Baur, Lecture Notes in Physics 365 (1990) 111; G.L. Kotkin, E.A. Kuraev, A. Schiller and V.G. Serbo, Phys. Rev. C59 (1999) 2734

[21] T. Aumann, P.F. Bortignon, H. Emling, Ann. Rev. Nucl. Part. Sci. 48 (1998) 282; C.A. Bertulani and V.Yu. Ponomarev, Phys. Reports 321 (1999) 139.

[22] C.A. Bertulani, L.F. Canto and M.S. Hussein, Phys. Rep. 226 (1993) 282; T. Glasmacher, Ann. Rev. Nucl. Part. Sci. 48 (1998) 1.

[23] S.A. Austin and G.F. Bertsch, Scientific American 272 (1995) 62; P. G. Hansen, A. S. Jensen, and B. Jonson, Ann. Rev. Nucl. Part. Sci. 45, 591 (1995).

[24] G. Baur, C.A. Bertulani and D.M. Kalassa, Nucl. Phys. A550 (1992) 527; K. Ieki, et al., Phys. Rev. Lett. 70 (1993) 730; G.F. Bertsch and C.A. Bertulani, Nucl. Phys. A556 (1993) 136; Phys. Rev. C49 (1994) 2834; H. Esbensen, G.F. Bertsch and C.A. bertulani, Nucl. Phys. A581 (1995) 107

[25] M.S.Hussein, R.A.Rego and C.A. Bertulani, Phys. Reports 201 (1991) 279; C.A. Bertulani, P. Lotti and H. Sagawa, Phys. Rev. C57 (1998) 217

[26] D.D. Clayton, Principles of Stellar Evolution and Nucleosynthesis, McGraw-Hill, New York, 1968; C. Rolfs and W.S. Rodney, Cauldrons in the Cosmos, Chicago Press, Chicago, 1988

[27] N. Iwasa, et al., Phys. Rev. Lett. 83 (1999) 2910

[28] A.J. Baltz, M.J. Rhoades-Brown, and J. Weneser, Phys. Rev E54 (1996) 4233; A.J. Baltz, C. Chasman and S.N. White, Nucl. Instrum. Meth, A417 (1998) 1; S.N. White, Nucl. Instrum. Meth. A409 (1998) 618

[29] M. Chiu, A. Denisov, E. Garcia, J. Katzy, M. Murray, S. White and Z. Xu,

[30] G. Baur and L.G. Ferreira Filho, Nucl. Phys. A518 (1990) 786; N. Cahn and J.D. Jackson, 
Phys. Rev. D42 (1990) 3690

[31] C.G. Roldão and A.A. Natale, Phys. Rev. C61 (2000) 064907; K.A. Chikin et al., e-print nucl-th/0002028

[32] G. Baur, K. Hencken and D. Trautmann, J. Phys. G24 (1998) 1657

[33] M. Grabiak, B. Müller, W. Greiner and P. Koch, J. Phys. G15 (1989) L25; E. Papageorgiu, Phys. Rev. D40 (1989) 92

[34] S.R. Klein and J. Nystrand, RHIC-STAR Note 1998/347; S. Klein, e-print physics/0012021

[35] S.R. Klein and J. Nystrand, Phys. Rev. C56, 014903 (1999)

[36] F. Dydak et al., Nucl. Phys. B118 (1977) 1; P.C. Petersen et. al., Phys. Rev. Lett., 57 (1986) 949

[37] Yu.M. Antipov et al., Phys. Rev. D36 (1987) 21

[38] Yu.M. Antipov et al., Phys. Lett. B121 (1983) 445; Z. Phys. C26 (1985) 495

[39] S. Klein and E. Scannapieco, RHIC-STAR Note 1997/243, "Two-photon physics with STAR"; G. Baur et al., LHC-CMS Note 2000/060, "Heavy ion physics programme in CMS"; H. Takai (for the ATLAS experiment), private communication

TABLE I. Two-photon meson production at RHIC and at LHC. Masses are in MeV, decay widths in $\mathrm{keV}$, and cross sections in mb. The cross sections are for $\gamma \gamma$ and pomeron-pomeron $(\mathcal{P} \mathcal{P})$ exchange processes, respectively.

\begin{tabular}{lllllll}
\hline \hline \hline Meson & $\mathrm{M}$ & $\Gamma_{X \rightarrow \gamma \gamma}$ & $R H I C_{\gamma \gamma}$ & $L H C_{\gamma \gamma}$ & $R H I C_{\mathcal{P P}}$ & $L H C_{\mathcal{P P}}$ \\
\hline$\pi^{0}$ & 135 & $8 \times 10^{-3}$ & 7.1 & 40 & 0.05 & 0.367 \\
$\eta$ & 547 & 0.463 & 1.5 & 17 & 0.038 & 0.355 \\
$\eta^{\prime}$ & 958 & 4.3 & 1.1 & 22 & 0.04 & 0.405 \\
\hline \hline \hline
\end{tabular}


TABLE II. One-photon vector meson production cross sections in mb at RHIC and at LHC.

\begin{tabular}{lll}
\hline \hline Meson & & \\
& RHIC & LHC \\
\hline$\rho^{0}$ & 590 & \\
$\omega$ & 59 & 5200 \\
$\omega$ & 39 & 490 \\
$\phi$ & 0.29 & 460 \\
$J / \Psi$ & 32 \\
\hline \hline
\end{tabular}

